Article

\title{
Solvability of the Boussinesq Approximation for Water Polymer Solutions
}

\author{
Mikhail A. Artemov (iD) and Evgenii S. Baranovskii * $\mathbb{D}$ \\ Department of Applied Mathematics, Informatics and Mechanics, Voronezh State University, \\ 394018 Voronezh, Russia \\ * Correspondence: esbaranovskii@gmail.com
}

Received: 22 June 2019; Accepted: 5 July 2019; Published: 10 July 2019

\begin{abstract}
We consider nonlinear Boussinesq-type equations that model the heat transfer and steady viscous flows of weakly concentrated water solutions of polymers in a bounded three-dimensional domain with a heat source. On the boundary of the flow domain, the impermeability condition and a slip condition are provided. For the temperature field, we use a Robin boundary condition corresponding to the classical Newton law of cooling. By using the Galerkin method with special total sequences in suitable function spaces, we prove the existence of a weak solution to this boundary-value problem, assuming that the heat source intensity is bounded. Moreover, some estimates are established for weak solutions.
\end{abstract}

Keywords: boundary-value problem; existence theorem; weak solutions; Boussinesq equations; heat transfer; water polymer solutions; second-grade fluids; slip boundary condition

\section{Introduction and Problem Formulation}

In this work, we examine the solvability of a boundary-value problem for the Boussinesq approximation describing steady-state flows of weakly concentrated water polymer solutions $[1,2]$ in a sufficiently regular bounded domain $\Omega \subset \mathbb{R}^{3}$ with a heat source, under the assumption that on solid walls of $\Omega$ the impermeability condition, a vorticity-slip condition and a Robin-type temperature boundary condition are valid:

$$
\begin{gathered}
\sum_{i=1}^{3} u_{i} \frac{\partial \vec{u}}{\partial x_{i}}-v \Delta \vec{u}-\alpha \sum_{i=1}^{3} u_{i} \frac{\partial \Delta \vec{u}}{\partial x_{i}}+\operatorname{grad} p=T \vec{g} \quad \text { in } \Omega, \\
\operatorname{div} \vec{u}=0 \quad \text { in } \Omega, \\
\sum_{i=1}^{3} u_{i} \frac{\partial T}{\partial x_{i}}-k \Delta T=\psi(\vec{x}, T) \quad \text { in } \Omega, \\
\left.\vec{u} \cdot \vec{n}\right|_{\partial \Omega}=0, \quad(\operatorname{curl} \vec{u}) \times\left.\vec{n}\right|_{\partial \Omega}=\overrightarrow{0}, \quad \frac{\partial T}{\partial \vec{n}}+\left.\beta T\right|_{\partial \Omega}=0 .
\end{gathered}
$$

Let us briefly describe the various terms in this system: the symbols $\vec{u}$ and $p$ stand for the velocity and the pressure, respectively, $T$ denotes the temperature field, the parameter $v>0$ represents the viscosity, $\alpha>0$ is the relaxation viscosity, $\psi$ denotes the heat source intensity, $\vec{g}=(0,0,-g)$ is the gravitational acceleration, $k>0$ is the thermal conductivity, $\beta>0$ is the Robin coefficient, and $\vec{n}$ is the unit outward normal to the surface $\partial \Omega$. The divergence, the gradient and the Laplacian $\Delta$ are taken with respect to the Cartesian coordinates $x_{1}, x_{2}, x_{3}$.

Notice that Equations (1) and (2) without the temperature term $T$ represent simplified equations of a stationary isothermal flow of the second grade fluid [3] (see also the book [4] for more details). 
Obviously, in the case when $\alpha=0$, we formally recover the stationary Navier-Stokes-Boussinesq system with a heat source, but, in what follows, we always assume that $\alpha>0$. As shown in [5-7], the parameter $\alpha$ must be non-negative, in view of thermodynamic restrictions.

It is worth pointing out that there is a vast amount of literature on the model of weakly concentrated water polymer solutions and its various modifications, including the so-called Kelvin-Voigt equations that describe viscous fluid flows in which, after the instantaneous removal of stresses, the velocity does not vanish instantaneously but decays exponentially. Starting with the pioneering series of works by A.P. Oskolkov [8-14], there is an ever growing list of contributions. An interested reader can see the papers [15-28]; this list is by no means exhaustive, but gives a number of current mathematical results obtained for these types of viscoelastic fluids.

In the majority of works, isothermal flows are investigated, although from the point of view of applications in engineering, the analysis of heat transfer in polymer flows is no less important than the studying of hydrodynamic fields. Motivated by this, we consider problem (1)-(4). The present paper continues the investigations initiated in the articles [10,29], where the thermal convection is studied for a simplified version of the model of water polymer solutions. Namely, the authors of these papers focus on the Kelvin-Voigt-Boussinesq equations, which have a lower order (for partial derivatives with respect to the space variables) compared with the equations considered herein.

Under the assumption that the intensity of the heat source is bounded, by the Galerkin method with special total sequences in suitable function spaces, we show the existence of a weak solution to boundary-value problem (1)-(4) and derive some estimates for the norms of the velocity and temperature fields.

\section{Preliminaries: Notations and Function Spaces}

For Banach spaces $E_{1}$ and $E_{2}$, by $\mathcal{L}\left(E_{1}, E_{2}\right)$, we denote the space of all linear and continuous operators from $E_{1}$ to $E_{2}$. The space $\mathcal{L}\left(E_{1}, E_{2}\right)$ is equipped with the norm

$$
\|A\|_{\mathcal{L}\left(E_{1}, E_{2}\right)} \stackrel{\text { def }}{=} \sup _{\|w\|_{E_{1}} \neq 0} \frac{\|A(w)\|_{E_{2}}}{\|w\|_{E_{1}}} .
$$

As usual, $C(\bar{\Omega})$ denotes the space of all continuous functions $w: \bar{\Omega} \rightarrow \mathbb{R}$.

We shall use the classical notation and results [30] for the Lebesgue spaces $L^{q}(\Omega), q \geq 1$, and the Sobolev spaces: $H^{m}(\Omega) \stackrel{\text { def }}{=} W^{m, 2}(\Omega), m \in\{1,2, \ldots\}$. By bold face letters, we denote the corresponding spaces of vector functions, e.g., $\mathbf{L}^{q}(\Omega) \stackrel{\text { def }}{=} L^{q}(\Omega)^{3}, \mathbf{H}^{1}(\Omega) \stackrel{\text { def }}{=} H^{1}(\Omega)^{3}$, etc.

Let us introduce the following divergence-free subspaces:

$$
\begin{aligned}
\mathbf{J}_{\vec{n}}^{1}(\Omega) & \stackrel{\text { def }}{=}\left\{\vec{v} \in \mathbf{H}^{1}(\Omega): \quad \operatorname{div} \vec{v}=0,\left.\quad \vec{v} \cdot \vec{n}\right|_{\partial \Omega}=0\right\}, \\
\mathbf{J}_{\vec{n}, \text { curl }}^{2}(\Omega) \stackrel{\text { def }}{=}\left\{\vec{v} \in \mathbf{H}^{2}(\Omega):\right. & \left.\operatorname{div} \vec{v}=0,\left.\quad \vec{v} \cdot \vec{n}\right|_{\partial \Omega}=0, \quad(\operatorname{curl} \vec{u}) \times\left.\vec{n}\right|_{\partial \Omega}=\overrightarrow{0}\right\}, \\
\mathbf{J}_{\vec{n}, \text { curl }}^{3}(\Omega) & \stackrel{\operatorname{def}}{=}\left\{\vec{v} \in \mathbf{H}^{3}(\Omega): \quad \operatorname{div} \vec{v}=0,\left.\quad \vec{v} \cdot \vec{n}\right|_{\partial \Omega}=\left.\Delta \vec{v} \cdot \vec{n}\right|_{\partial \Omega}=0, \quad(\operatorname{curl} \vec{u}) \times\left.\vec{n}\right|_{\partial \Omega}=\overrightarrow{0}\right\} .
\end{aligned}
$$

By $\mathbf{X}_{\alpha}(\Omega)$ denote the space consisting of vector functions from $\mathbf{J}_{\vec{n} \text {, curl }}^{2}(\Omega)$ with scalar product defined according to the relation

$$
(\vec{v}, \vec{w})_{\mathbf{X}_{\alpha}(\Omega)} \stackrel{\text { def }}{=} \int_{\Omega} \operatorname{curl} \vec{v} \cdot \operatorname{curl} \vec{w} d x+\alpha \int_{\Omega} \Delta \vec{v} \cdot \Delta \vec{w} d x .
$$

It follows from ([16] § 2) that the scalar product $(\cdot, \cdot) \mathbf{X}_{\alpha}(\Omega)$ is well defined and the associated norm $\|\cdot\|_{\mathbf{X}_{\alpha}(\Omega)} \stackrel{\text { def }}{=}(\cdot, \cdot)_{\mathbf{X}_{\alpha}(\Omega)}^{1 / 2}$ is equivalent to the standard $\mathbf{H}^{2}$-norm. 
Finally, by $Y_{\beta}(\Omega)$ denote the space consisting of functions from $H^{1}(\Omega)$ with the following scalar product and norm:

$$
(S, Q)_{Y_{\beta}(\Omega)} \stackrel{\text { def }}{=} \int_{\Omega} \operatorname{grad} S \cdot \operatorname{grad} Q d x+\beta \int_{\partial \Omega} S Q d \sigma, \quad\|S\|_{Y_{\beta}(\Omega)} \stackrel{\text { def }}{=}(S, S)_{Y_{\beta}(\Omega)}^{1 / 2} \cdot
$$

It can easily be checked that the scalar product $(\cdot, \cdot)_{Y_{\beta}(\Omega)}$ is well defined and the norm $\|\cdot\|_{Y_{\beta}(\Omega)}$ is equivalent to the standard $H^{1}$-norm.

\section{Weak Formulation of Problem (1)-(4) and Main Results}

We propose the problem of determining the velocity field $\vec{u} \in \mathbf{X}_{\alpha}(\Omega)$ and the temperature $T \in Y_{\beta}(\Omega)$ that satisfy the system of equations:

$$
\begin{aligned}
& -\sum_{i=1}^{3} \int_{\Omega} u_{i} \vec{u} \cdot \frac{\partial \vec{v}}{\partial x_{i}} d x-v \int_{\Omega} \Delta \vec{u} \cdot \vec{v} d x+\alpha \sum_{i=1}^{3} \int_{\Omega} u_{i} \Delta \vec{u} \cdot \frac{\partial \vec{v}}{\partial x_{i}} d x=\int_{\Omega} T \vec{g} \cdot \vec{v} d x \\
& \sum_{i=1}^{3} \int_{\Omega} u_{i} \frac{\partial T}{\partial x_{i}} S d x+k \int_{\Omega} \operatorname{grad} T \cdot \operatorname{grad} S d x+k \beta \int_{\partial \Omega} T S d \sigma=\int_{\Omega} \psi(\vec{x}, T) S d x
\end{aligned}
$$

for any pair $(\vec{v}, S)$ from the space $\mathbf{J}_{\vec{n}}^{1}(\Omega) \times H^{1}(\Omega)$.

Definition 1. A solution $(\vec{u}, T) \in \mathbf{X}_{\alpha}(\Omega) \times Y_{\beta}(\Omega)$ to system (5) and (6) is called a weak solution of boundary value problem (1)-(4).

The following theorem gives the main result of this paper.

Theorem 1. Let us assume that

(i) the function $\psi(\cdot, \xi): \Omega \rightarrow \mathbb{R}$ is measurable for every $\xi \in \mathbb{R}$;

(ii) the function $\psi(\vec{x}, \cdot): \mathbb{R} \rightarrow \mathbb{R}$ is continuous for almost every $\vec{x} \in \Omega$;

(iii) there exists a positive constant $\psi_{0}$ such that $|\psi(\vec{x}, \xi)| \leq \psi_{0}$ for almost every $\vec{x} \in \Omega$ and every $\xi \in \mathbb{R}$.

Then, boundary value problem (1)-(4) has at least one weak solution $(\vec{u}, T)$ such that

$$
\begin{aligned}
\|\vec{u}\|_{\mathbf{X}_{\alpha}(\Omega)} & \leq \frac{g \psi_{0} M_{2}^{2}(\Omega, \beta)\left(M_{1}(\Omega, \alpha)+\alpha^{1 / 2}\right)(\operatorname{meas}(\Omega))^{1 / 2}}{k v}, \\
\|T\|_{\gamma_{\beta}(\Omega)} \leq & \leq \frac{\psi_{0} M_{2}(\Omega, \beta)(\operatorname{meas}(\Omega))^{1 / 2}}{k},
\end{aligned}
$$

with

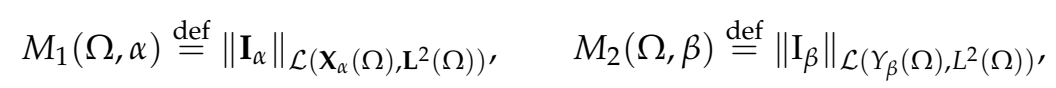

where $\mathbf{I}_{\alpha}: \mathbf{X}_{\alpha}(\Omega) \rightarrow \mathbf{L}^{2}(\Omega)$ and $\mathrm{I}_{\beta}: Y_{\beta}(\Omega) \rightarrow L^{2}(\Omega)$ are embedding operators.

\section{Proof of Theorem 1}

To construct a weak solution of problem (1)-(4), we shall use the Galerkin method. Let us take a sequence $\left\{\vec{w}^{j}\right\}_{j=1}^{\infty}$ from the space $\mathbf{J}_{\vec{n}}^{3}$, curl $(\Omega)$ such that $\left\{\vec{w}^{j}\right\}_{j=1}^{\infty}$ is total in $\mathbf{J}_{\vec{n} \text {, curl }}^{3}(\Omega)$ and

$$
\left(\vec{w}^{i}, \vec{w}^{j}\right)_{\mathbf{X}_{\alpha}(\Omega)}=\delta_{i j}
$$

where $\delta_{i j}$ is the Kronecker symbol. In addition, fix a sequence $\left\{S^{j}\right\}_{j=1}^{\infty}$ that is an orthonormal basis of $Y_{\beta}(\Omega)$.

For an arbitrary fixed number $N \in\{1,2, \ldots\}$, we consider the $2 N$-dimensional auxiliary problem: 
Find a vector $\vec{h}_{\lambda}^{N}=\left(a_{N 1}, \ldots, a_{N N}, b_{N 1}, \ldots, b_{N N}\right) \in \mathbb{R}^{2 N}$ such that

$$
\begin{aligned}
-\lambda \sum_{i=1}^{3} \int_{\Omega} & u_{i}^{N} \vec{u}^{N} \cdot \frac{\partial\left(\vec{w}^{j}-\alpha \Delta \vec{w}^{j}\right)}{\partial x_{i}} d x-v \int_{\Omega} \Delta \vec{u}^{N} \cdot\left(\vec{w}^{j}-\alpha \Delta \vec{w}^{j}\right) d x \\
& \quad+\lambda \alpha \sum_{i=1}^{3} \int_{\Omega} u_{i}^{N} \Delta \vec{u}^{N} \cdot \frac{\partial\left(\vec{w}^{j}-\alpha \Delta \vec{w}^{j}\right)}{\partial x_{i}} d x=\lambda \int_{\Omega} T^{N} \vec{g}^{\prime} \cdot\left(\vec{w}^{j}-\alpha \Delta \vec{w}^{j}\right) d x, \quad j=1, \ldots, N,
\end{aligned}
$$

$$
\begin{aligned}
& \lambda \sum_{i=1}^{3} \int_{\Omega} u_{i}^{N} \frac{\partial T^{N}}{\partial x_{i}} S^{j} d x+k \int_{\Omega} \operatorname{grad} T^{N} \cdot \operatorname{grad} S^{j} d x+k \beta \int_{\partial \Omega} T^{N} S^{j} d \sigma=\lambda \int_{\Omega} \psi\left(\vec{x}, T^{N}\right) S^{j} d x, \quad j=1, \ldots, N, \\
& \vec{u}^{N}(\vec{x}) \stackrel{\text { def }}{=} \sum_{j=1}^{N} a_{N j} \vec{w}^{j}(\vec{x}), \quad T^{N}(\vec{x}) \stackrel{\text { def }}{=} \sum_{j=1}^{N} b_{N j} S^{j}(\vec{x}),
\end{aligned}
$$

where $\lambda$ is a parameter, $\lambda \in[0,1]$.

Our immediate goal is to obtain a priori estimates for solutions to problem (9)-(11). Let a vector $\vec{h}_{\lambda}^{N}=\left(a_{N 1}, \ldots, a_{N N}, b_{N 1}, \ldots, b_{N N}\right)$ be a solution of problem (9)-(11) under a fixed parameter $\lambda \in[0,1]$. It is easy to see that

$$
\left|\vec{h}_{\lambda}^{N}\right|^{2}=\sum_{j=1}^{N} a_{N j}^{2}+\sum_{j=1}^{N} b_{N j}^{2}=\left\|\vec{u}^{N}\right\|_{\mathbf{X}_{\alpha}(\Omega)}^{2}+\left\|T^{N}\right\|_{Y_{\beta}(\Omega)}^{2} .
$$

Therefore, we wish to find estimates for the norms $\left\|\vec{u}^{N}\right\|_{\mathbf{x}_{\alpha}(\Omega)}$ and $\left\|T^{N}\right\|_{Y_{\beta}(\Omega)}$.

Let us multiply Equation (9) by $a_{N j}$ and add the results for $j=1, \ldots, N$. Then, we get

$$
\begin{aligned}
& -\lambda \sum_{i=1}^{3} \int_{\Omega} u_{i}^{N} \vec{u}^{N} \cdot \frac{\partial\left(\vec{u}^{N}-\alpha \Delta \vec{u}^{N}\right)}{\partial x_{i}} d x-v \int_{\Omega} \Delta \vec{u}^{N} \cdot\left(\vec{u}^{N}-\alpha \Delta \vec{u}^{N}\right) d x \\
& +\lambda \alpha \sum_{i=1}^{3} \int_{\Omega} u_{i}^{N} \Delta \vec{u}^{N} \cdot \frac{\partial\left(\vec{u}^{N}-\alpha \Delta \vec{u}^{N}\right)}{\partial x_{i}} d x=\lambda \int_{\Omega} T^{N} \vec{g} \cdot\left(\vec{u}^{N}-\alpha \Delta \vec{u}^{N}\right) d x
\end{aligned}
$$

This equality can be rewritten as follows:

$$
\begin{aligned}
-\lambda \underbrace{\sum_{i=1}^{3} \int_{\Omega} u_{i}^{N}\left(\vec{u}^{N}-\alpha \Delta \vec{u}^{N}\right) \cdot \frac{\partial\left(\vec{u}^{N}-\alpha \Delta \vec{u}^{N}\right)}{\partial x_{i}} d x}_{Q_{1}}-v \int_{\Omega} \Delta \vec{u}^{N} \cdot\left(\vec{u}^{N}-\alpha \Delta \vec{u}^{N}\right) d x & =\lambda \int_{\Omega} T^{N} \vec{g}^{N} \cdot\left(\vec{u}^{N}-\alpha \Delta \vec{u}^{N}\right) d x
\end{aligned}
$$

Note that the term $Q_{1}$ vanishes. Indeed, using integration by parts, we find that

$$
\begin{aligned}
Q_{1} & =\frac{1}{2} \sum_{i=1}^{3} \int_{\Omega} u_{i}^{N} \frac{\partial}{\partial x_{i}}\left|\vec{u}^{N}-\alpha \Delta \vec{u}^{N}\right|^{2} d x \\
& =\frac{1}{2} \sum_{i=1}^{3} \int_{\partial \Omega} u_{i}^{N} n_{i}\left|\vec{u}^{N}-\alpha \Delta \vec{u}^{N}\right|^{2} d \sigma-\frac{1}{2} \sum_{i=1}^{3} \int_{\Omega} \frac{\partial u_{i}^{N}}{\partial x_{i}}\left|\vec{u}^{N}-\alpha \Delta \vec{u}^{N}\right|^{2} d x \\
& =\frac{1}{2} \int_{\partial \Omega}\left(\vec{u}^{N} \cdot \vec{n}\right)\left|\vec{u}^{N}-\alpha \Delta \vec{u}^{N}\right|^{2} d \sigma-\frac{1}{2} \int_{\Omega}\left(\operatorname{div} \vec{u}^{N}\right)\left|\vec{u}^{N}-\alpha \Delta \vec{u}^{N}\right|^{2} d x \\
& =0 .
\end{aligned}
$$


Therefore, we have

$$
-v \int_{\Omega} \Delta \vec{u}^{N} \cdot\left(\vec{u}^{N}-\alpha \Delta \vec{u}^{N}\right) d x=\lambda \int_{\Omega} T^{N} \vec{g} \cdot\left(\vec{u}^{N}-\alpha \Delta \vec{u}^{N}\right) d x .
$$

Taking into account the relation

$$
-\int_{\Omega} \Delta \vec{v} \cdot \vec{w} d x=\int_{\Omega} \operatorname{curl} \vec{v} \cdot \operatorname{curl} \vec{w} d x, \quad \forall \vec{v} \in \mathbf{J}_{\vec{n}, \operatorname{curl}}^{2}(\Omega), \vec{w} \in \mathbf{H}^{1}(\Omega),
$$

we can rewrite (13) as

$$
v \int_{\Omega}\left|\operatorname{curl} \vec{u}^{N}\right|^{2} d x+v \alpha \int_{\Omega}\left|\Delta \vec{u}^{N}\right|^{2} d x=\lambda \int_{\Omega} T^{N} \vec{g} \cdot \vec{u}^{N} d x-\lambda \alpha \int_{\Omega} T^{N} \vec{g} \cdot \Delta \vec{u}^{N} d x .
$$

It follows from this equality that

$$
\begin{aligned}
v\left\|\vec{u}^{N}\right\|_{\boldsymbol{X}_{\alpha}(\Omega)}^{2} & =v \int_{\Omega}\left|\operatorname{curl} \vec{u}^{N}\right|^{2} d x+v \alpha \int_{\Omega}\left|\Delta \vec{u}^{N}\right|^{2} d x \\
& =\lambda \int_{\Omega} T^{N} \vec{g} \cdot \vec{u}^{N} d x-\lambda \alpha \int_{\Omega} T^{N} \vec{g} \cdot \Delta \vec{u}^{N} d x,
\end{aligned}
$$

whence, using the Cauchy-Bunyakovsky-Schwarz inequality, the estimates $0 \leq \lambda \leq 1$ and

$$
\left\|\vec{u}^{N}\right\|_{\mathbf{L}^{2}(\Omega)} \leq M_{1}(\Omega, \alpha)\left\|\vec{u}^{N}\right\|_{\mathbf{X}_{\alpha}(\Omega)}, \quad\left\|T^{N}\right\|_{L^{2}(\Omega)} \leq M_{2}(\Omega, \beta)\left\|T^{N}\right\|_{\gamma_{\beta}(\Omega)},
$$

we derive

$$
\begin{aligned}
v\left\|\vec{u}^{N}\right\|_{\mathbf{X}_{\alpha}(\Omega)}^{2} \leq & g\left(\int_{\Omega}\left|T^{N}\right|^{2} d x\right)^{1 / 2}\left(\int_{\Omega}\left|\vec{u}^{N}\right|^{2} d x\right)^{1 / 2} \\
& +g \alpha^{1 / 2}\left(\int_{\Omega}\left|T^{N}\right|^{2} d x\right)^{1 / 2}\left(\alpha \int_{\Omega}\left|\Delta \vec{u}^{N}\right|^{2} d x\right)^{1 / 2} \\
= & g\left\|T^{N}\right\|_{L^{2}(\Omega)}\left\|\vec{u}^{N}\right\|_{\mathbf{L}^{2}(\Omega)}+g \alpha^{1 / 2}\left\|T^{N}\right\|_{L^{2}(\Omega)}\left\|\alpha^{1 / 2} \Delta \vec{u}^{N}\right\|_{\mathbf{L}^{2}(\Omega)} \\
\leq & g M_{2}(\Omega, \beta)\left(M_{1}(\Omega, \alpha)+\alpha^{1 / 2}\right)\left\|T^{N}\right\|_{\gamma_{\beta}(\Omega)}\left\|\vec{u}^{N}\right\|_{\mathbf{X}_{\alpha}(\Omega)}
\end{aligned}
$$

and hence

$$
\left\|\vec{u}^{N}\right\|_{\mathbf{X}_{\alpha}(\Omega)} \leq \frac{g M_{2}(\Omega, \beta)\left(M_{1}(\Omega, \alpha)+\alpha^{1 / 2}\right)}{v}\left\|T^{N}\right\|_{\gamma_{\beta}(\Omega)} .
$$

Next, we multiply Equation (10) by $b_{N j}$ and add the results for $j=1, \ldots, N$; this gives

$$
\lambda \underbrace{\sum_{i=1}^{3} \int_{\Omega} u_{i}^{N} \frac{\partial T^{N}}{\partial x_{i}} T^{N} d x}_{Q_{2}}+k \int_{\Omega}\left|\operatorname{grad} T^{N}\right|^{2} d x+k \beta \int_{\partial \Omega}\left|T^{N}\right|^{2} d \sigma=\lambda \int_{\Omega} \psi\left(\vec{x}, T^{N}\right) T^{N} d x .
$$

Applying integration by parts, it can easily be checked that the term $Q_{2}$ vanishes. Indeed,

$$
\begin{aligned}
Q_{2} & =\frac{1}{2} \sum_{i=1}^{3} \int_{\Omega} u_{i}^{N} \frac{\partial}{\partial x_{i}}\left|T^{N}\right|^{2} d x \\
& =\frac{1}{2} \sum_{i=1}^{3} \int_{\partial \Omega} u_{i}^{N} n_{i}\left|T^{N}\right|^{2} d \sigma-\frac{1}{2} \sum_{i=1}^{3} \int_{\Omega} \frac{\partial u_{i}^{N}}{\partial x_{i}}\left|T^{N}\right|^{2} d x \\
& =\int_{\partial \Omega}\left(\vec{u}^{N} \cdot \vec{n}\right)\left|T^{N}\right|^{2} d \sigma-\frac{1}{2} \int_{\Omega}\left(\operatorname{div} \vec{u}^{N}\right)\left|T^{N}\right|^{2} d x \\
& =0 .
\end{aligned}
$$


Therefore, we arrive at the equality

$$
k \int_{\Omega}\left|\operatorname{grad} T^{N}\right|^{2} d x+k \beta \int_{\partial \Omega}\left|T^{N}\right|^{2} d \sigma=\lambda \int_{\Omega} \psi\left(\vec{x}, T^{N}\right) T^{N} d x,
$$

whence, using the Cauchy-Bunyakovsky-Schwarz inequality, condition (iii), the second inequality of (14), and $0 \leq \lambda \leq 1$, we derive

$$
\begin{aligned}
k\left\|T^{N}\right\|_{\gamma_{\beta}(\Omega)}^{2} & =\lambda \int_{\Omega} \psi\left(\vec{x}, T^{N}\right) T^{N} d x \\
& \leq\left(\int_{\Omega}\left|\psi\left(\vec{x}, T^{N}\right)\right|^{2} d x\right)^{1 / 2}\left(\int_{\Omega}\left|T^{N}\right|^{2} d x\right)^{1 / 2} \\
& \leq \psi_{0}(\operatorname{meas}(\Omega))^{1 / 2}\left\|T^{N}\right\|_{L^{2}(\Omega)} \\
& \leq \psi_{0}(\operatorname{meas}(\Omega))^{1 / 2} M_{2}(\Omega, \beta)\left\|T^{N}\right\|_{\gamma_{\beta}(\Omega)} .
\end{aligned}
$$

This yields that

$$
\left\|T^{N}\right\|_{\gamma_{\beta}(\Omega)} \leq \frac{\psi_{0} M_{2}(\Omega, \beta)(\operatorname{meas}(\Omega))^{1 / 2}}{k} .
$$

Combining (15) and (16), we get

$$
\left\|\vec{u}^{N}\right\|_{\mathbf{X}_{\alpha}(\Omega)} \leq \frac{g \psi_{0}(\operatorname{meas}(\Omega))^{1 / 2} M_{2}^{2}(\Omega, \beta)\left(M_{1}(\Omega, \alpha)+\alpha^{1 / 2}\right)}{k v} .
$$

Finally, using (12), (16) and (17), we obtain the following a priori estimate

$$
\left|\vec{h}_{\lambda}^{N}\right|^{2} \leq \frac{g^{2} \psi_{0}^{2} \operatorname{meas}(\Omega) M_{2}^{4}(\Omega, \beta)\left(M_{1}(\Omega, \alpha)+\alpha^{1 / 2}\right)^{2}}{k^{2} v^{2}}+\frac{\psi_{0}^{2} M_{2}^{2}(\Omega, \beta) \operatorname{meas}(\Omega)}{k^{2}},
$$

which is independent of $N$ as well as $\lambda$.

Next, we define the operators $\mathbf{A}, \mathbf{G}_{\lambda}: \mathbb{R}^{2 N} \rightarrow \mathbb{R}^{2 N}$ as follows:

- $\quad$ for each $m \in\{1, \ldots, N\}$, we set

$$
\begin{gathered}
A_{m}\left(a_{N 1}, \ldots, a_{N N}, b_{N 1}, \ldots, b_{N N}\right) \stackrel{\text { def }}{=} v \int_{\Omega} \operatorname{curl} \vec{u}^{N} \cdot \operatorname{curl} \vec{w}^{m} d x+v \alpha \int_{\Omega} \Delta \vec{u}^{N} \cdot \Delta \vec{w}^{m} d x, \\
\underbrace{}_{\lambda m}\left(a_{N 1}, \ldots, a_{N N}, b_{N 1}, \ldots, b_{N N}\right) \stackrel{\text { def }}{=}-\lambda \sum_{i=1}^{3} \int_{\Omega} u_{i}^{N} \vec{u}^{N} \cdot \frac{\partial\left(\vec{w}^{m}-\alpha \Delta \vec{w}^{m}\right)}{\partial x_{i}} d x \\
+\lambda \alpha \sum_{i=1}^{3} \int_{\Omega} u_{i}^{N} \Delta \vec{u}^{N} \cdot \frac{\partial\left(\vec{w}^{m}-\alpha \Delta \vec{w}^{m}\right)}{\partial x_{i}} d x \\
-\lambda \int_{\Omega} T^{N} \vec{g}^{\prime} \cdot\left(\vec{w}^{m}-\alpha \Delta \vec{w}^{m}\right) d x
\end{gathered}
$$

- for each $m \in\{N+1, \ldots, 2 N\}$, we set

$$
\begin{gathered}
A_{m}\left(a_{N 1}, \ldots, a_{N N}, b_{N 1}, \ldots, b_{N N}\right) \stackrel{\text { def }}{=} k \int_{\Omega} \operatorname{grad} T^{N} \cdot \operatorname{grad} S^{m-N} d x+k \beta \int_{\partial \Omega} T^{N} \cdot S^{m-N} d \sigma, \\
G_{\lambda m}\left(a_{N 1}, \ldots, a_{N N}, b_{N 1}, \ldots, b_{N N}\right) \stackrel{\text { def }}{=} \lambda \sum_{i=1}^{3} \int_{\Omega} u_{i}^{N} \frac{\partial T^{N}}{\partial x_{i}} S^{m-N} d x-\lambda \int_{\Omega} \psi\left(\vec{x}, T^{N}\right) S^{m-N} d x,
\end{gathered}
$$

where $\left(a_{N 1}, \ldots, a_{N N}, b_{N 1}, \ldots, b_{N N}\right)$ is an arbitrary vector from space $\mathbb{R}^{2 N}$, while functions $\vec{u}^{N}$ and $T^{N}$ are defined by (11). 


\section{Letting}

$\mathbf{F}\left(a_{N 1}, \ldots, a_{N N}, b_{N 1}, \ldots, b_{N N}, \lambda\right) \stackrel{\text { def }}{=} \mathbf{A}\left(a_{N 1}, \ldots, a_{N N}, b_{N 1}, \ldots, b_{N N}\right)+\mathbf{G}_{\lambda}\left(a_{N 1}, \ldots, a_{N N}, b_{N 1}, \ldots, b_{N N}\right)$,

we note that the Galerkin system (9)-(11) is equivalent to the following equation

$$
\mathbf{F}\left(a_{N 1}, \ldots, a_{N N}, b_{N 1}, \ldots, b_{N N}, \lambda\right)=\overrightarrow{0} \in \mathbb{R}^{2 N},
$$

where $\lambda \in[0,1]$.

It is worth pointing out that

$$
\mathbf{F}\left(a_{N 1}, \ldots, a_{N N}, b_{N 1}, \ldots, b_{N N}, 0\right)=\mathbf{A}\left(a_{N 1}, \ldots, a_{N N}, b_{N 1}, \ldots, b_{N N}\right)
$$

and the operator $\mathbf{A}$ is an isomorphism due to the relation

$$
\mathbf{A}\left(a_{N 1}, \ldots, a_{N N}, b_{N 1}, \ldots, b_{N N}\right)=\left(v a_{N 1}, \ldots, v a_{N N}, k b_{N 1}, \ldots, k b_{N N}\right) .
$$

Then, an application of Proposition A1 (see the Appendix A) together with a priori estimate (18) allows us to state that problem (9)-(11) is solvable for any $N \in\{1,2, \ldots\}$ and $\lambda \in[0,1]$.

Let $\left\{\vec{u}_{*}^{N}\right\}_{N=1}^{\infty}$ and $\left\{T_{*}^{N}\right\}_{N=1}^{\infty}$ be sequences of functions that satisfy (9) and (10) with the parameter $\lambda=1$, i.e.,

$$
\begin{aligned}
& -\sum_{i=1}^{3} \int_{\Omega} u_{* i}^{N} \vec{u}_{*}^{N} \cdot \frac{\partial\left(\vec{w}^{j}-\alpha \Delta \vec{w}^{j}\right)}{\partial x_{i}} d x-v \int_{\Omega} \Delta \vec{u}_{*}^{N} \cdot\left(\vec{w}^{j}-\alpha \Delta \vec{w}^{j}\right) d x \\
& \quad+\alpha \sum_{i=1}^{3} \int_{\Omega} u_{* i}^{N} \Delta \vec{u}_{*}^{N} \cdot \frac{\partial\left(\vec{w}^{j}-\alpha \Delta \vec{w}^{j}\right)}{\partial x_{i}} d x=\int_{\Omega} T_{*}^{N} \vec{g} \cdot\left(\vec{w}^{j}-\alpha \Delta \vec{w}^{j}\right) d x, \quad j=1, \ldots, N, \\
& \sum_{i=1}^{3} \int_{\Omega} u_{* i}^{N} \frac{\partial T_{*}^{N}}{\partial x_{i}} S^{j} d x+k \int_{\Omega} \operatorname{grad} T_{*}^{N} \cdot \operatorname{grad} S^{j} d x+k \beta \int_{\partial \Omega} T_{*}^{N} S^{j} d \sigma \\
& \quad=\int_{\Omega} \psi\left(\vec{x}, T_{*}^{N}\right) S^{j} d x, \quad j=1, \ldots, N .
\end{aligned}
$$

It follows from estimates (16) and (17) that the set $\left\{\vec{u}_{*}^{N}\right\}_{N=1}^{\infty}$ is bounded in the space $\mathbf{X}_{\alpha}(\Omega)$ and the set $\left\{T_{*}^{N}\right\}_{N=1}^{\infty}$ is bounded in the space $Y_{\beta}(\Omega)$. Hence, there exists a vector function $\vec{u}_{0}$ from the space $\mathbf{X}_{\alpha}(\Omega)$ and a function $T_{0}$ from the space $Y_{\beta}(\Omega)$ such that $\vec{u}_{*}^{N^{\prime}}$ converges to $\vec{u}_{0}$ weakly in $\mathbf{X}_{\alpha}(\Omega)$ and $T_{*}^{N^{\prime}}$ converges to $T_{0}$ weakly in $Y_{\beta}(\Omega)$, for some subsequence $N^{\prime} \rightarrow \infty$. Without loss of generality, we can assume that

$$
\begin{aligned}
& \lim _{N \rightarrow \infty} \vec{u}_{*}^{N}=\vec{u}_{0} \text { weakly in } \mathbf{X}_{\alpha}(\Omega), \\
& \lim _{N \rightarrow \infty} T_{*}^{N}=T_{0} \text { weakly in } Y_{\beta}(\Omega) .
\end{aligned}
$$

Using standard compactness results for the Sobolev spaces (see, e.g., [30] Chap. 6), we derive that the space $\mathbf{X}_{\alpha}(\Omega)$ is compactly imbedded into $\mathbf{C}(\bar{\Omega})$ and the space $Y_{\beta}(\Omega)$ is compactly imbedded into $L^{q}(\Omega)$ when $1 \leq q<6$. Therefore, it follows from (22) and (23) that

$$
\begin{aligned}
& \lim _{N \rightarrow \infty} \vec{u}_{*}^{N}=\vec{u}_{0} \text { strongly in } \mathbf{C}(\bar{\Omega}), \\
& \lim _{N \rightarrow \infty} T_{*}^{N}=T_{0} \text { strongly in } L^{q}(\Omega),
\end{aligned}
$$

where $1 \leq q<6$. 
Furthermore, by using the theorem of M.A. Krasnoselskii on a superposition operator (see the Appendix A, Proposition A2), we conclude from conditions (i)-(iii) and (25) that

$$
\lim _{N \rightarrow \infty} \psi\left(\cdot, T_{*}^{N}(\cdot)\right)=\psi\left(\cdot, T_{0}(\cdot)\right) \text { strongly in } L^{2}(\Omega) \text {. }
$$

Fix an arbitrary number $j \in\{1,2, \ldots\}$. Then, letting $m \rightarrow \infty$ in (20) and (21), we get

$$
\begin{aligned}
& -\sum_{i=1}^{3} \int_{\Omega} u_{0 i} \vec{u}_{0} \cdot \frac{\partial\left(\vec{w}^{j}-\alpha \Delta \vec{w}^{j}\right)}{\partial x_{i}} d x-v \int_{\Omega} \Delta \vec{u}_{0} \cdot\left(\vec{w}^{j}-\alpha \Delta \vec{w}^{j}\right) d x \\
& \quad+\alpha \sum_{i=1}^{3} \int_{\Omega} u_{0 i} \Delta \vec{u}_{0} \cdot \frac{\partial\left(\vec{w}^{j}-\alpha \Delta \vec{w}^{j}\right)}{\partial x_{i}} d x=\int_{\Omega} T_{0} \vec{g} \cdot\left(\vec{w}^{j}-\alpha \Delta \vec{w}^{j}\right) d x, \\
& \quad \sum_{i=1}^{3} \int_{\Omega} u_{0 i} \frac{\partial T_{0}}{\partial x_{i}} S^{j} d x+k \int_{\Omega} \operatorname{grad} T_{0} \cdot \operatorname{grad} S^{j} d x+k \beta \int_{\partial \Omega} T_{0} S^{j} d \sigma=\int_{\Omega} \psi\left(\vec{x}, T_{0}\right) S^{j} d x .
\end{aligned}
$$

Of course, in this passage-to-limit procedure, we used all the convergence results (22)-(26). It follows from ([16] §2) that the mapping $\mathcal{R}_{\alpha}$ defined by

$$
\mathcal{R}_{\alpha}: \mathbf{J}_{\vec{n}, \text { curl }}^{3}(\Omega) \rightarrow \mathbf{J}_{\vec{n}}^{1}(\Omega), \quad \mathcal{R}_{\alpha}(\vec{v}) \stackrel{\text { def }}{=} \vec{v}-\alpha \Delta \vec{v}
$$

is an isomorphism. Therefore, the sequence $\left\{\vec{w}^{j}-\alpha \Delta \vec{w}^{j}\right\}_{j=1}^{\infty}$ is total in the space $\mathbf{J}_{\vec{n}}^{1}(\Omega)$. This fact is a key tool in our proof. Indeed, due to this property, equality (27) remains valid if we replace $\vec{w}^{j}-\alpha \Delta \vec{w}^{j}$ with an arbitrary vector function $\vec{v}$ from $\mathbf{J}_{\vec{n}}^{1}(\Omega)$. In addition, since the set $\left\{S^{j}\right\}_{j=1}^{\infty}$ is total in $H^{1}(\Omega)$, we see that (28) is true with an arbitrary function $S$ from $H^{1}(\Omega)$ instead of $S^{j}$. Thus, we have established that the pair $\left(\vec{u}_{0}, T_{0}\right)$ is a weak solution of problem (1)-(4).

Moreover, in view of estimates (16) and (17), we obviously have the inequalities (7) and (8) with $\vec{u}=\vec{u}_{0}$ and $T=T_{0}$. The proof of Theorem 1 is complete.

\section{Conclusions}

In this paper, we considered nonlinear Boussinesq-type equations describing the heat transfer and steady viscous flows of weakly concentrated water solutions of polymers in a bounded three-dimensional domain with sufficiently smooth boundary. We proved the existence of weak solutions in suitable function classes. Besides, some estimates for weak solutions are obtained in terms of the data of this model.

Author Contributions: Writing-Original draft M.A.A.; methodology, E.S.B.; Writing-review and editing, E.S.B. Funding: This research received no external funding.

Conflicts of Interest: The authors declare no conflict of interest.

\section{Appendix A}

For the reader's convenience, let us state an important generalization of the Brouwer fixed-point theorem, which is used in our proof.

Proposition A1. Suppose $\mathbf{B}_{r} \stackrel{\text { def }}{=}\left\{\vec{x} \in \mathbb{R}^{d}:|\vec{x}|<r\right\}$ and a continuous operator $\mathbf{F}: \overline{\mathbf{B}}_{r} \times[0,1] \rightarrow \mathbb{R}^{d}$ satisfies the following conditions:

- $\mathbf{F}(\vec{x}, \lambda) \neq \overrightarrow{0}$ for any $(\vec{x}, \lambda) \in \partial \mathbf{B}_{r} \times[0,1]$;

- $\mathbf{F}(\vec{x}, 0)=\mathcal{A} \vec{x}$ for any $\vec{x} \in \overline{\mathbf{B}}_{r}$, where $\mathcal{A}: \mathbb{R}^{d} \rightarrow \mathbb{R}^{d}$ is an isomorphism.

Then, for any $\lambda \in[0,1]$, the equation $\mathbf{F}(\vec{x}, \lambda)=\overrightarrow{0}$ has at least one solution $\vec{x}_{\lambda}$, which belong to the ball $\mathbf{B}_{r}$. 
This proposition can be proved by methods of the topological degree theory (see, e.g., [31]).

In addition, recall the well-known theorem of M.A. Krasnoselskii on a superposition operator acting in Lebesgue spaces.

Proposition A2. Let $f: \Omega \times \mathbb{R} \rightarrow \mathbb{R}$ be a function such that

- the function $f(\cdot, \xi): \Omega \rightarrow \mathbb{R}$ is measurable for every $\xi \in \mathbb{R}$;

- the function $f(\vec{x}, \cdot): \mathbb{R} \rightarrow \mathbb{R}$ is continuous for almost every $\vec{x} \in \Omega$;

- there exist constants $q_{1}, q_{2} \geq 1, C>0$ and a function $\phi \in L^{q_{2}}(\Omega)$ such that the following inequality holds

$$
|f(\vec{x}, \xi)| \leq \phi(\vec{x})+C|\xi|^{q_{1} / q_{2}},
$$

for almost every $\vec{x} \in \Omega$ and every $\xi \in \mathbb{R}$.

Under these hypotheses, the superposition operator $\Phi_{f}$ defined by

$$
\Phi_{f}: L^{q_{1}}(\Omega) \rightarrow L^{q_{2}}(\Omega), \quad \Phi_{f}(w)(\vec{x}) \stackrel{\text { def }}{=} f(\vec{x}, w(\vec{x}))
$$

is a bounded and continuous mapping.

The proof of this result can be found in the book ([32] Chap. 1).

\section{References}

1. Pavlovskir, V.A. On the theoretical description of weak water solutions of polymers. Dokl. Akad. Nauk SSSR 1971, 200, 809-812. (In Russian)

2. Amfilokhiev, V.B.; Voitkunskiı̌, Y.I.; Mazaeva, N.P.; Khodorkovskiř, Y.S. Flows of polymer solutions under convective accelerations. Tr. Leningrad. Korabl. Inst. 1975, 96, 3-9. (In Russian)

3. Rivlin, R.S.; Ericksen, J.L. Stress-deformation relations for isotropic materials. J. Ration. Mech. Anal. 1955, 4, 323-425. [CrossRef]

4. Cioranescu, D.; Girault, V.; Rajagopal, K.R. Mechanics and Mathematics of Fluids of the Differential Type; Springer: Cham, Switzerland, 2016. [CrossRef]

5. Ting, T.-W. Certain non-steady flows of second order fluids. Arch. Ration. Mech. Anal. 1963, 14, 1-26. [CrossRef]

6. Coleman, B.D.; Duffin, R.J.; Mizel, V. Instability, uniqueness, and non-existence theorems for the equation $u_{t}=u_{x x}-u_{x t x}$ on a strip. Arch. Ration. Mech. Anal. 1965, 19, 100-116. [CrossRef]

7. Dunn, J.E.; Fosdick, R.L. Thermodynamics, stability, and boundedness of fluids of complexity 2 and fluids of second grade. Arch. Ration. Mech. Anal. 1974, 56, 191-252. [CrossRef]

8. Oskolkov, A.P. On the uniqueness and solvability in the large of the boundary-value problems for the equations of motion of aqueous solutions of polymers. Zap. Nauchn. Semin. LOMI 1973, 38, 98-136.

9. Oskolkov, A.P. A nonstationary quasilinear system with a small parameter, regularizing a system of Navier-Stokes equations. J. Sov. Math. 1976, 6, 51-57. [CrossRef]

10. Oskolkov, A.P. Some nonstationary linear and quasilinear systems occurring in the investigation of the motion of viscous fluids. J. Math. Sci. 1978, 10, 299-335. [CrossRef]

11. Oskolkov, A.P. Theory of nonstationary flows of Kelvin-Voigt fluids. J. Sov. Math. 1985, 28, 751-758. [CrossRef]

12. Oskolkov, A.P. Initial-boundary value problems for the equations of motion of Kelvin-Voigt fluids and Oldroyd fluids. Proc. Steklov Inst. Math. 1989, 179, 137-182.

13. Oskolkov, A.P. Nonlocal problems for the equations of motion of Kelvin-Voight fluids. J. Math. Sci. 1995, 75, 2058-2077. [CrossRef]

14. Oskolkov, A.P. The initial boundary-value problem with a free surface condition for the penalized equations of aqueous solutions of polymers. J. Math. Sci. 1997, 83, 320-326. [CrossRef]

15. Sviridyuk, G.A.; Sukacheva, T.G. On the solvability of a nonstationary problem describing the dynamics of an incompressible viscoelastic fluid. Math. Notes 1998, 63, 388-395. [CrossRef] 
16. Ladyzhenskaya, O.A. On the global unique solvability of some two-dimensional problems for the water solutions of polymers. J. Math. Sci. 2000, 99, 888-897. [CrossRef]

17. Ladyzhenskaya, O.A. In memory of A.P. Oskolkov. J. Math. Sci. 2000, 99, 799-801. [CrossRef]

18. Sviridyuk, G.A.; Plekhanova, M.V. An optimal control problem for the Oskolkov equation. Differ. Equ. 2002, 38, 1064-1066. [CrossRef]

19. Kuz'min, M.Y. On Boundary-Value Problems for Some Models of Hydrodynamics with Slip Conditions at the Boundary; Candidate's Dissertation in Mathematics and Physics: Voronezh, Russia, 2007.

20. Garcia-Luengo, J.; Marin-Rubio, P.; Real, J. Pullback attractors for three-dimensional non-autonomous Navier-Stokes-Voigt equations. Nonlinearity 2012, 25, 905-930. [CrossRef]

21. Baranovskii, E.S. An optimal boundary control problem for the motion equations of polymer solutions. Sib. Adv. Math. 2014, 24, 159-168. [CrossRef]

22. Baranovskii, E.S. Flows of a polymer fluid in domain with impermeable boundaries. Comput. Math. Math. Phys. 2014, 54, 1589-1596. [CrossRef]

23. Guo, Y.; Cheng, S.; Tang, Y. Approximate Kelvin-Voigt fluid driven by an external force depending on velocity with distributed delay. Discrete Dyn. Nat. Soc. 2015, 2015, 1-9. [CrossRef]

24. Bozhkov, Y.D.; Pukhnachev, V.V. Group analysis of equations of motion of aqueous solutions of polymers. Dokl. Phys. 2015, 60, 77-80. [CrossRef]

25. Baranovskii, E.S. Mixed initial-boundary value problem for equations of motion of Kelvin-Voigt fluids. Comput. Math. Math. Phys. 2016, 56, 1363-1371. [CrossRef]

26. Cao, J.; Qin, Y. Pullback attractors of 2D incompressible Navier-Stokes-Voight equations with delay. Math. Meth. Appl. Sci. 2017, 40, 6670-6683. [CrossRef]

27. Baranovskii, E.S. Global solutions for a model of polymeric flows with wall slip. Math. Meth. Appl. Sci. 2017, 40, 5035-5043. [CrossRef]

28. Yang, X.; Feng, B.; de Souza, T.M.; Wang, T. Long-time dynamics for a non-autonomous Navier-Stokes-Voigt equation in Lipschitz domains. Discret. Contin. Dyn. Syst. Ser. B 2018, 22, 1-24. [CrossRef]

29. Sviridyuk, G.A. Solvability of a problem of the thermoconvection of a viscoelastic incompressible fluid. Soviet. Math. 1990, 34, 80-86.

30. Adams, R.A.; Fournier, J.J.F. Sobolev Spaces, Vol. 40 of Pure and Applied Mathematics; Academic Press: Amsterdam, The Netherlands, 2003.

31. Lloyd, N.G. Degree Theory; Cambridge University Press: Cambridge, UK, 1978.

32. Krasnoselskii, M.A. Topological Methods in the Theory of Nonlinear Integral Equations; Pergamon Press: New York, NY, USA, 1964.

(C) 2019 by the authors. Licensee MDPI, Basel, Switzerland. This article is an open access article distributed under the terms and conditions of the Creative Commons Attribution (CC BY) license (http:/ / creativecommons.org/licenses/by/4.0/). 